\title{
Cognitive Load Theory and Complex Learning: Recent Developments and Future Directions
}

\author{
Jeroen J. G. van Merriënboer \\ Educational Technology Expertise Center, Open University of the Netherlands \\ John Sweller \\ School of Education, University of New South Wales
}

Traditionally, Cognitive Load Theory (CLT) has focused on instructional methods to decrease extraneous cognitive load so that available cognitive resources can be fully devoted to learning. This article strengthens the cognitive base of CLT by linking cognitive processes to the processes used by biological evolution. The article discusses recent developments in CLT related to the current view in instructional design that real-life tasks should be the driving force for complex learning. First, the complexity, or intrinsic cognitive load, of such tasks is often high so that new methods are needed to manage cognitive load. Second, complex learning is a lengthy process requiring learners' motivational states and levels of expertise development to be taken into account. Third, this perspective requires more advanced methods to measure expertise and cognitive load so that instruction can be flexibly adapted to individual learners' needs. Experimental studies are reviewed to illustrate these recent developments. Guidelines for future research are provided.

Educational Psychology Review, 17, 147-177

http://www.springerlink.com/content/v637454300v27622/

(C) 2005 Springer, all rights reserved 